\title{
Rating of Physiotherapy Student Clinical Performance in A Paediatric Setting: Is It Possible to Gain Assessor Consistency?
}

\section{Tessa Fulton}

Queensland Children's Hospital

\section{Kerry Myatt}

Queensland Children's Hospital

\section{Garry W Kirwan}

Metro South Health

Courtney R Clark ( $\nabla$ courtney.clark@griffith.edu.au )

Griffith University

Megan Dalton

Australian Catholic University

\section{Research Article}

Keywords: Clinical education, Physiotherapy, Paediatrics, Assessment, APP

Posted Date: February 25th, 2021

DOI: https://doi.org/10.21203/rs.3.rs-228209/v1

License: (a) (i) This work is licensed under a Creative Commons Attribution 4.0 International License. Read Full License 


\section{Title:}

Rating of physiotherapy student clinical performance in a paediatric setting: is it possible to gain assessor consistency?

\section{Author List:}

1. Tessa Fulton (Corresponding Author). Physiotherapy Department, Queensland Children's Hospital, South Brisbane QLD, Australia 4001. Tessa.Fulton@health.qld.gov.au

2. Kerry Myatt. Physiotherapy Department, Queensland Children's Hospital, South Brisbane QLD, Australia 4001. Kerry.Myatt@health.qld.gov.au

3. Dr Garry W Kirwan: Physiotherapy Department, QEII Jubilee Hospital, Metro South Health, Coopers Plains QLD, Australia 4109. Menzies Health Institute, School of Allied Health Sciences, Griffith University, Australia 4222.

Garry.Kirwan@health.qld.gov.au

4. Courtney R Clark. Griffith Institute for Educational Research, Griffith University, Gold Coast Campus, Australia 4222. courtney.clark@griffith.edu.au

5. Associate Professor Megan Dalton. School of Physiotherapy, Australian Catholic University Brisbane Australia.Megan.dalton@acu.edu.au

\section{Corresponding author:}

Courtney Clark

Griffith Institute for Educational Research, Griffith University

1 Parklands Drive, Southport, QLD, 4215, Australia

$+61755527564$

Courtney.clark@grifith.edu.au 


\begin{abstract}
Background:

During workplace based clinical placements, best practice in assessment dictates that students should expect consistency between assessors rating their performance. To assist clinical educators (CEs) to provide a consistent assessment of physiotherapy student performance, nine paediatric vignettes depicting various standards of student performance, as assessed by the Assessment of Physiotherapy Practice (APP), were developed. The project aimed to evaluate the consistency of physiotherapy educators assessing student competence in a paediatric setting using video vignettes alongside the APP.
\end{abstract}

\title{
Methods:
}

Thirty-six CEs, with minimum 3-years clinical experience and had supervised a student within the past year, were sent three videos at four-week intervals. Videos depicted the same clinical scenario, however student performance varied with each video. Consistency among raters was assessed using percentage agreement to establish reliability.

\section{Results:}

The vignettes were assessed a combined total of 60 times. Across scenarios, percentage agreement at the not adequate level was $100 \%$, and combining adequate or better, percentage agreement was $>86 \%$. The study demonstrated strong consensus when comparing not adequate to adequate or better student performance. Importantly, no student performance scripted as not adequate was passed by any assessor.

\section{Conclusions:}


Experienced educators demonstrate consistency in identifying a not adequate from adequate or better performance when assessing a one-off student performance using the APP. These validated video vignettes will be a valuable training tool to improve educator consistency when assessing student performance in paediatric physiotherapy.

\section{Keywords}

Clinical education, Physiotherapy, Paediatrics, Assessment, APP 


\section{Background}

Quality clinical education in paediatric physiotherapy is integral to the development of competent health professional graduates. In physiotherapy programs across Australia and New Zealand, students are assessed on their ability to deliver entry-level physiotherapy services to the paediatric population across a variety of clinical settings. The minimally competent standard is defined within the Physiotherapy Practice Threshold Statements (1). However, to assess performance effectively, consistency independent of the clinical area and setting is essential to maintain grade integrity $(2,3)$.

Grade integrity defines a grade as "representing the quality, breadth and depth of the level of achievement a student reaches" (4). Essentially the grade applied to a student performance is representative of the actual performance and so that grade is able to accurately determine the level of competence of the student. Competence is an ongoing state and therefore assessment needs to include actual performance as well as the demonstrated ability to adapt to change and seek new information (5). Within the paediatric setting, physiotherapy students are assessed using the Assessment of Physiotherapy Practice (APP) (6); (7). The APP defines an adequate on the global rating scale (GRS) as the minimal acceptable standard for an entry level physiotherapist. A non-adequate rating indicates the student did not demonstrate the minimal acceptable standard and a good or excellent rating will indicate that the student performances at a level above the minimum standard. However, anecdotally clinicians within the paediatric setting report additional challenges to the interpretation of the APP due to the nuances of the paediatric environment. Furthermore, there is a lack of resources and training to support this clinical setting.

Evidence supports the benefit of consensus moderation to deliver consistent, accurate and effective assessment (8). This is supported by the fact that learning resources related to 
assessment using the APP are available for other clinical settings. A study conducted by (9) demonstrated the potential variability that may exist in clinical assessment and how training and resources using video vignettes can augment assessment practices.

In response to a gap in the literature, the primary aim of this study is to determine the level of consistency among paediatric clinical educators when assessing a student performance using the APP. The secondary aim was to collate qualitative data on assessment decisions to aid in the development of training resources to improve student assessment in the physiotherapy paediatric setting.

\section{Methods}

Ethical approval was granted by The Human Research Ethics Committee of Queensland Children's Hospital (protocol number - HREC/16/QRCH/362) and Griffith University (protocol number 2016/941) prior to the study's commencement. All methods were carried out in accordance with the relevant guidelines and regulations. Informed consent was obtained from all participants as per the ethics guidelines.

Development of the vignettes:

Three paediatric scenarios representing the core area of neurodevelopment across three age ranges, infant, toddler and adolescent, were developed and scripted into performances that depicted a 'not adequate', 'adequate' and 'good to excellent' student performance based on the APP GRS. The scenarios included an infant with failure to thrive, a pre-school aged child with developmental coordination disorder and an adolescent with Cerebral Palsy. An expert panel of clinical educators $(n=9)$, experienced in one of the three areas of paediatrics mentioned, was convened to conduct face and content validation. Each panel member reviewed each of the three performance levels from their clinical area of expertise $(n=3$ 
scripts per panel member). Panel feedback on the level of performance, clinical authenticity and accuracy of the script was collected and recommendations to improve the face and content validity were collected for incorporation into the final scripts.

Once agreement was reached for all nine scripts, each video was filmed using a standardised actor to portray the student physiotherapist. Children who portrayed patients during filming were known to the project team and consented to participate. This included one child with a known neurological condition and two children who were typically developing. Clinical staff from Queensland Children's Hospital played the role of clinical educator and parent. During the filming of each scenario, the authors were present to direct each scene to ensure adherence to the script. Each video was on average 18 mins in length.

Assessment of reliability

A purposive sample of physiotherapists currently providing paediatric clinical education in Australia were invited to participate in the study. The Inclusion criteria was a minimum three years clinical experience in paediatrics and 1-year experience in the clinical education of physiotherapy students. Participants who did not meet the inclusion criteria were excluded to ensure the sample population was familiar with the assessment of student performance using the APP

Paediatric physiotherapists identified as meeting the inclusion criteria were invited to participate via email correspondence. Participants were provided with a Participant Information sheet detailing their involvement including the nature of the study and the total time commitment. Consent to participate was achieved via an 'opt in' approach with a response to the sent email indicating consent to participate. Consent was confirmed from the participants prior to completing the study survey (Supplementary 1). Participants included in 
the study were allocated to a clinical scenario in their nominated area of expertise (infant, toddler or adolescent).

Following provision of consent and group allocation, participants were emailed detailed directions for reviewing the video vignette and completing the evaluation via Survey Monkey®. Each participant was sent a total of three videos vignettes, representing 'not adequate', 'adequate' and 'good to excellent', over a 12-week period of time. The vignettes were sent in a randomly allocated order to minimise bias. At the completion of the first vignette, participants were asked to provide demographic information in addition to a global rating scale based on the APP, key factors used to determine the global rating and feedback on video quality and clinical relevance that was collected for all three video vignettes. Vignettes were securely stored on Google Drive and distributed by email in the form of a closed link.

Participants were required to watch and complete a survey for three video vignettes in the same clinical area. A wash out period of four weeks between the sending of each video was selected to ensure that it was unlikely that participants would be able to recall specific information of the previously watched video (10), (11).

The video and survey links were closed two weeks after the initial email. After a 4 week wash out period, the same process was repeated with the second video with a new video and survey link. To maximize response, a reminder email was sent to all participants one week after the initial email. Furthermore, all participants who completed the study by watching all three videos were provided a financial incentive equating to \$50AUD.

Data Analysis:

Data from SurveyMonkey ${ }^{\circledR}$ was extracted as a comma-separated value (CSV) file and converted to Microsoft Excel ${ }^{\mathrm{TM}}$ (Microsoft Corporation, Redmond WA). The data was 
cleaned and separated into percentage agreement, demographic, key performance indicators (KPIs) and video vignette evaluation data. Percentage agreement was calculated for each of the nine videos using SPSS 21.0 software package ${ }^{\circledR}$ (SPSS Inc., Chicago, IL, USA). Key performance indicators for each video were collected for thematic analysis and divided into the seven domains on the APP (professional behaviour, communication, assessment, analysis and planning, intervention, evidence based practice and risk management).

\section{Results}

Participant Demographics:

Forty-three clinicians across various clinical settings were identified as meeting the inclusion criteria and invited to participate, of which 36 consented to participate. Four participants (11\%) withdrew from the study sighting time constraints $(n=3)$ and technical issues $(n=1)$ as primary reason. Twenty-seven (75\%) completed at least one video and 17 went on to complete all three videos assessments (47.2\%). In total, the suite of videos was viewed a total of 63 times for the purposes of data collection.

Responses were received from participants in four states of Australia (Queensland $=66 \%$, Victoria $=21 \%, \mathrm{NSW}=8 \%$, and Western Australia $=5 \%$ ). Most participants reported working in the public hospital setting $(60 \%)$, however there was representation from a variety of clinical settings as outlined in Figure 1 (Title: Primary clinical setting of participants; X: Percentage; Y: Primary clinical setting; Legend: Distribution of Primary clinical setting of respondents). $63 \%$ of participants worked in a metropolitan area, with $35 \%$ regional and $2 \%$ rural/remote. Participants reported a range of clinical experience in the field of paediatric physiotherapy, as well as their paediatric clinical education experience (Table 1). Ninety- 
seven percent of participants reported a confidence level of 'somewhat confident' or greater with $73 \%$ being confident or very confident in using the APP to assess student performance. 
Table 1 Participant characteristics

\begin{tabular}{|c|c|c|}
\hline Participant Demographics & Frequency (n) & Percent $(\%)$ \\
\hline \multicolumn{3}{|l|}{ Clinical Experience $(\mathrm{n}=60)$} \\
\hline $3-5$ years & 3 & 5 \\
\hline $6-8$ years & 13 & 21.66 \\
\hline $9-11$ years & 8 & 13.33 \\
\hline $12-15$ years & 7 & 11.66 \\
\hline More than 15 years & 29 & 48.33 \\
\hline \multicolumn{3}{|c|}{ Experience as an Educator $(\mathrm{n}=60)$} \\
\hline $1-3$ years & 13 & 21.66 \\
\hline $4-6$ years & 16 & 26.66 \\
\hline $7-9$ years & 9 & 15 \\
\hline $10-12$ years & 5 & 8.33 \\
\hline $12-14$ years & 3 & 5 \\
\hline More than 14 years & 14 & 23.33 \\
\hline \multicolumn{3}{|c|}{ Confidence in using the APP $(n=60)$} \\
\hline Not confident & 2 & 3.33 \\
\hline Somewhat confident & 15 & 25 \\
\hline Confident & 36 & 60 \\
\hline Very confident & 7 & 11.66 \\
\hline
\end{tabular}

APP-Assessment of Physiotherapy Practice 
Percentage Agreement:

A total of 63 complete responses were collected across the nine video vignettes and included for analysis.

Percentage agreement for the Infant, Toddler and Adolescent videos at the not adequate and good-excellent level achieved greater than the $75 \%$ agreement. In contrast, the adequate scenarios for the Infant, Toddler and Adolescent video failed to meet the $75 \%$ agreement level (Table 2). However, when analysing the adequate videos by comparing not adequate to a combined rating of adequate or better, percentage agreement increased to $>75 \%$ in all categories (Table 3). Only one assessor rated the adequate scenario for the Infant, Toddler and Adolescent as not adequate. 
Table 2: Scenarios and percentage agreement with APP GRS

\begin{tabular}{|c|c|c|c|c|c|c|c|}
\hline & & \multicolumn{5}{|c|}{ BLIND ASESSMENT } & \multirow[b]{2}{*}{ Agreement } \\
\hline & & Not Adequate & Adequate & Good & Excellent & & \\
\hline & Not Adequate & 4 & & 0 & 0 & 0 & $100 \%$ \\
\hline \multirow[t]{3}{*}{ INFANT } & Adequate & 0 & & 4 & 1 & 4 & $44.40 \%$ \\
\hline & Good-Excellent & 0 & & 0 & 3 & 6 & $100 \%$ \\
\hline & Not Adequate & $\overline{4}$ & & 0 & 0 & 0 & $100 \%$ \\
\hline \multirow[t]{3}{*}{ TODDLER } & Adequate & 0 & & 6 & 2 & 1 & $66.60 \%$ \\
\hline & Good-Excellent & 0 & & 0 & 5 & 3 & $100 \%$ \\
\hline & Not Adequate & 6 & & 0 & 0 & 0 & $100 \%$ \\
\hline \multirow[t]{2}{*}{ ADOLESCENT } & Adequate & 1 & & 0 & 4 & 2 & $0 \%$ \\
\hline & Good-Excellent & 0 & & 1 & 0 & 6 & $85.71 \%$ \\
\hline
\end{tabular}


Table 3: Adequate scenario percentage agreement with APP GRS

\begin{tabular}{|c|c|c|c|c|}
\hline & & $\begin{array}{l}\text { Not } \\
\text { Adequate }\end{array}$ & $\begin{array}{l}\text { Adequate or } \\
\text { better }\end{array}$ & Agreement \\
\hline \multirow{3}{*}{ 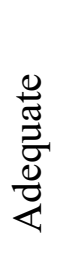 } & Infant & 0 & 9 & $100 \%$ \\
\hline & Toddler & 0 & 9 & $100 \%$ \\
\hline & Adolescent & 1 & 6 & $86 \%$ \\
\hline
\end{tabular}

Thematic analysis:

Participants were asked to provide three to five KPIs that they used to formulate their global rating of each video. Thematic analysis highlighted consistent themes across all nine videos and are outlined in Table 4. 
Table 4: Adequate performance indicators as commented by clinical educators

\begin{tabular}{|c|c|c|c|c|c|}
\hline \multicolumn{6}{|c|}{ Summary of Adequate performance indicators } \\
\hline Professional behaviour & Communication & Assessment & Analysis \& Planning & Intervention & Risk management \\
\hline \multirow[t]{3}{*}{$\begin{array}{l}\text { Could have been more } \\
\text { sensitive in discussing the } \\
\text { child's difficulties in front } \\
\text { of the child }\end{array}$} & $\begin{array}{l}\text { Communicated effectively } \\
\text { and appropriately }\end{array}$ & $\begin{array}{l}\text { Performed appropriate } \\
\text { assessment however } \\
\text { missed some important } \\
\text { information such as birth } \\
\text { history and milestones }\end{array}$ & $\begin{array}{l}\text { Interpretation of } \\
\text { assessment findings could } \\
\text { be improved }\end{array}$ & $\begin{array}{l}\text { When providing feedback } \\
\text { to mother, still uncertain } \\
\text { about what low muscle } \\
\text { tone is and its functional } \\
\text { impact }\end{array}$ & $\begin{array}{l}\text { Monitors patient/client } \\
\text { safety during assessment } \\
\text { and treatment }\end{array}$ \\
\hline & Provided clear instructions & $\begin{array}{l}\text { Required some prompting } \\
\text { with choice of assessment } \\
\text { items }\end{array}$ & $\begin{array}{l}\text { Needs to improve skills in } \\
\text { explaining assessment } \\
\text { findings to parent. }\end{array}$ & $\begin{array}{l}\text { Didn't fully address } \\
\text { mother's concerns or } \\
\text { questions or refer to other } \\
\text { allied health/ send program } \\
\text { to Kindy }\end{array}$ & \\
\hline & $\begin{array}{l}\text { Needed some prompting to } \\
\text { adapt communication to } \\
\text { appropriate level for the } \\
\text { child (demonstration of } \\
\text { task) }\end{array}$ & $\begin{array}{l}\text { Muscle tone test was a bit } \\
\text { rushed and head position } \\
\text { not monitored and } \\
\text { controlled. }\end{array}$ & & $\begin{array}{l}\text { Effective education of } \\
\text { patent and child }\end{array}$ & \\
\hline
\end{tabular}


Evaluation of video vignettes:

All participants either agreed (56\%) or strongly agreed (44\%) that the clinical scenario they viewed were realistic and believable. Most participants (98\%) reported that the clinical scenario was professional and well presented.

\section{Discussion}

The aim of the project was to undertake a reliability study for a suite of video vignettes depicting paediatric physiotherapy student performance based on the APP GRS. The study demonstrated acceptable consensus among participants at the not adequate and good to excellent level. However, at the adequate level there was insufficient exact agreement. If ratings of adequate and good-excellent were combined consensus once again reached an acceptable level when comparing 'not adequate' to 'adequate' or better.

In line with previous research investigating consensus in the adult clinical setting, the study showed strong agreement in rating the not adequate and the good-excellent student performance across all three clinical scenarios (9). This is relevant for clinical practice, particularly in relation to the level of agreement when rating the not adequate videos. Consistency in scoring students correctly using the GRS is essential for ensuring all preregistration students meet an adequate level of performance prior to entering the physiotherapy profession (7). In this study, all not adequate students were correctly identified by participants, and only one adequate performance received a not adequate rating.

There was insufficient consensus for the adequate video scenarios, which is a similar outcome to the previous adult video vignettes research (9). Student performance at both the 
not adequate and excellent levels is more easily recognised and rated by assessors whereas the variability in students performing at an adequate standard makes assessment at this level more difficult. Due to the relatively short length of the video performance (average length across all three scenarios was 18.35 minutes), and that educators were not able to observe students' repeat performances across multiple patients as they would do in traditional longitudinal clinical placements, participants may have missed important aspects of the student's performance and thus influenced their rating using the APP GRS. While use of short video vignettes of student performance is a recognised method used for consensus moderation and training of assessors to assess student performance during practical/OSCE examinations, determining if a student is performing at an adequate level in the clinical setting requires longitudinal assessment of performance $(12,13)$. It may be that for future training of clinical educators, multiple video vignettes scripted at the level of an adequate/passing performance are required to effectively calibrate assessors.

When adjusting the data in the adequate scenarios to compare an adequate or better versus a not adequate student performance, strong consensus was achieved. This indicates that it is more difficult to differentiate an adequate student performance from a good-excellent student performance.

\section{Conclusions}

Experienced educators demonstrated consistency in identifying a not adequate from adequate or better performance when assessing a one-off student performance using the APP. However, variability existed when assessing the 'adequate' performance with a lack of consensus in differentiating 'adequate' from 'good/excellent'.

The resources developed for this body of research are freely available online (https://www.applinkup.com/Resources.aspx). A corresponding training package is available 
to be utilized by physiotherapists and universities for paediatric clinical educator support and training, student learning and development, and delivery of training and workshops. 


\section{References}

1. Australia PBo, Zealand PBoN. Physiotherapy practice thresholds in Australia and Aotearoa New Zealand https://physiocouncil.com.au/media/1020/physiotherapy-board-physiotherapypractice-thresholds-in-australia-and-aotearoa-new-zealand-6.pdf: Australian Physiothertapy Council; 2015 [

2. Trede F, Smith, M. Workplace educators' interpretations of their assessment practices: A view through a critical practice lens. Assessment and Evaluation in Higher Education. 2014;39(2):154-67.

3. Sadler DR. Grade integrity and the representation of academic achievement. Studies in Higher Education. 2009;34(7):807-26.

4. Sadler DR. Academic freedom, achievement standards and professional identity. Quality in Higher Education. 2011;17(1):85-100.

5. Epstein R. Assessment in Medical Education. N Engl J Med. 2007;356:387-96.

6. Dalton M, Davidson, M, Keating, J The Assessment of Physiotherapy Practice (APP) is a valid measure of professional competence of physiotherapy students: a cross-sectional study with Rasch analysis. Journal of Physiotherapy. 2011;57(4):239-46.

7. Dalton M, Davidson, M, Keating, JL. The Assessment of Physiotherapy Practice (APP) is a reliable measure of professional competence of physiotherapy students: a reliability study. Journal of Physiotherapy. 2012;58(1):49-56.

8. Price M. Assessment standards: the role of communities of practice and the scholarship of assessment. Assessment \& Evaluation in Higher Education. 2005;30(3):215-30.

9. Kirwan GW, Clark CR, Dalton M. Rating of physiotherapy student clinical performance: is it possible to gain assessor consistency? BMC medical education. 2019;19(1):32.

10. Litwin MS. How to measure survey reliability and validity: Sage publications; 1995.

11. Streiner L, \& Norman, G, R. Health measurement scales: a practical guide to their development and use: Oxford: Oxford University Press; 1995.

12. van der Vleuten $\mathrm{C}$. Validity of final examinations in undergraduate medical training. Bmj. 2000;321(7270):1217-9.

13. Rethans JJ, Norcini J, Baron-Maldonado M, Blackmore D, Jolly B, LaDuca T, et al. The relationship between competence and performance: implications for assessing practice performance. Medical education. 2002;36(10):901-9. 


\section{List of abbreviations}

CE - Clinical Educator

APP - Assessment of Physiotherapy Practice

GRS - Global Rating Scale

CSV - Comma Separated Value

KPI - Key Performance Indicator

\section{Declarations:}

Trial registration, ethics approval and consent to participate: Ethical approval was granted by The Human Research Ethics Committee of Queensland Children's Hospital (protocol number - HREC/16/QRCH/362) and Griffith University (protocol number 2016/941) prior to the study's commencement. Subsequent amendments were approved including expansion of the study to a multi-centre study as well as to provide participants with a small incentive to maximise response rate. Site Specific Approvals were completed for participating sites. All methods were carried out in accordance with relevant guidelines and regulations. Informed consent was obtained from all subjects as per the ethics approval.

Consent for publication:

Not applicable

Availability of data and materials:

The datasets used and/or analysed during the current study are available from the corresponding author on reasonable request. 
Competing interests:

The authors declare that they have no competing interests.

Funding:

This project was funded via the Directors' of Physiotherapy Services Queensland (DOPSQ) Physiotherapy Clinical Education and Training Initiative (CETI) with support from the Allied Health Professions' Office of Queensland (AHPOQ). Funds were generated from the Physiotherapy Pre-Registration Clinical Placement Agreement which facilitates a partnership between the 16 Hospital and Health Services, Mater Health Services (Brisbane) and the 6 tertiary education providers offering physiotherapy programs in Queensland.

DOPSQ, through the Student Education and Training Standing Group (SET SG), has a vision to work together to provide high quality student clinical education for a work-ready and sustainable Physiotherapy workforce. CETI funds have been allocated to this project to help facilitate the achievement of this vision.

Authors' contributions:

TF, KM, GK, CC \& MD collaboratively contributed to the conception, design, data collection, analysis and manuscript development for the study presented.

Acknowledgments:

The Research Team would like to acknowledge the contribution of several individuals and organisations to this project: 
- Kellie Stockton - Director of Physiotherapy, Division of Clinical Support, Children's Health Queensland HHS

- Mr Paul Barber - Director of Physiotherapy, Division of Allied Health, Darling Downs HHS. Project Sponsor representing Directors of Physiotherapy Services Queensland Student Education and Training Standing Group (DOPSQ SET SG)

- This project was a collaboration between Children's Health Queensland (CHQ), Griffith University (GU) and Australian Catholic University (ACU). The project was developed to build on a previous project led by Griffith University in which video vignettes were developed in the adult setting and underwent an Australia wide validation process. GU provided in kind support for the filming and editing of the video vignettes. 
Figures

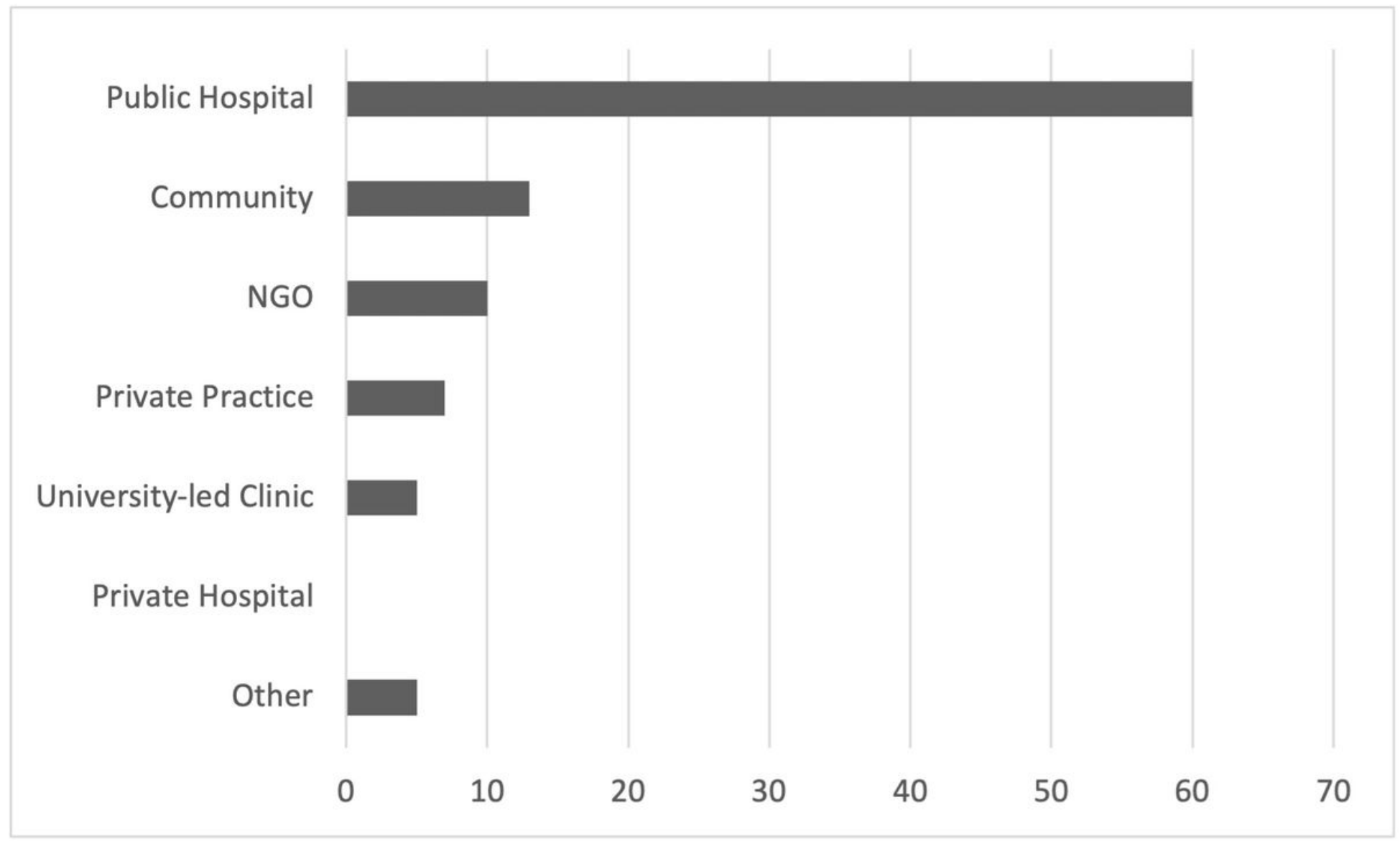

\section{Figure 1}

Primary clinical setting of participants; $X$ : Percentage; $Y$ : Primary clinical setting; Distribution of Primary clinical setting of respondents

\section{Supplementary Files}

This is a list of supplementary files associated with this preprint. Click to download.

- Fultonetal2021ConsensusinratingpaediatricassessmentusingtheAPPSupplementary.pdf 